\title{
Fracking en el sur de Mendoza: riesgos, incertidumbres y resistencias en contexto de una mega-sequía
}

Lucrecia Wagner

Instituto Argentino de Nivología, Glaciología y Ciencias Ambientales (IANIGLA).

Mendoza, Argentina.

CONICET. Mendoza, Argentina.

Recibido: 11 de mayo de 2021. Aceptado: 20 de septiembre de 2021.

\begin{abstract}
Resumen
En el año 2017, coincidiendo con la formación geológica Vaca Muerta -ubicada en las provincias argentinas de Mendoza, Neuquén, Río Negro y La Pampa-, comenzó a utilizarse en la provincia de Mendoza la técnica de fracking para la explotación de hidrocarburos no convencionales. En Mendoza, las resistencias a la megaminería basadas en la defensa del agua, un bien escaso y muy preciado, han sido de las más exitosas y trascendentes a nivel nacional. Sin embargo, el fracking tuvo una rápida autorización acompañada por la flexibilización de la legislación ambiental y un duro cuestionamiento a las voces críticas por parte del gobierno provincial. Ante este panorama, en el presente trabajo analizaremos cómo, a diferencia de lo acontecido en torno a la minería a gran escala, en el caso del fracking la protección del agua fue descuidada y la oposición social criminalizada. Por su parte, movimientos socioambientales y otros actores sociales, especialmente del sector científico-académico, han colocado en el debate público la situación de "mega-sequía" que atraviesa la provincia, la escasa evaluación de los riesgos y la incertidumbre generada por estas falencias. Una incertidumbre que se incrementa por el inicio de nuevas actividades extractivas que generan controversias y contradicciones con las políticas de cambio climático y con los objetivos de avanzar hacia una transición energética.
\end{abstract}

PALABRAS CLAVE: FRACKING. VACA MUERTA. SEQUÍA. RIESGO. INCERTIDUMBRE.

\section{Fracking in the south of Mendoza: risks, uncertainties and resistance in the context of a mega drought}

\begin{abstract}
In 2017, corresponding with the Vaca Muerta geological formation -located in the Argentinian provinces of Mendoza, Neuquén, Río Negro y La Pampa-, the fracking technique began to be used in the province of Mendoza for the exploitation of unconventional hydrocarbons. In Mendoza, the resistance to mega-mining based on the
\end{abstract}


defense of water, a scarce and highly valued good, has been one of the most successful and transcendent at the national level. However, fracking had a rapid authorization accompanied by the flexibilization of environmental legislation and a harsh questioning of the critical voices by the provincial government. Given this panorama, in this paper we will analyze how, unlike what happened around large-scale mining, in the case of fracking water protection was neglected and social opposition criminalized. For their part, socio-environmental movements and other social actors, especially from the scientific-academic sector, have placed in the public debate the situation of "megadrought" that the province is going through, the scarce evaluation of the risks and the uncertainty generated by these shortcomings. An uncertainty that is increased by the start of new extractive activities that generate controversies and contradictions with climate change policies and with the objectives of moving towards an energy transition.

KEYWORDS: FRACKING. VACA MUERTA. DROUGHT. RISK. UNCERTAINTY.

PALAVRAS-CHAVE: FRACKING. VACA MUERTA. SECA. RISCO. INCERTEZA.

\section{Introducción}

Argentina posee una de las mayores reservas de petróleo y gas no convencional del mundo. Se trata de la formación geológica "Vaca Muerta" ubicada en las provincias de Neuquén, Río Negro, Mendoza y La Pampa. Este trabajo abordará la problemática generada por el uso de la técnica de fractura hidráulica para la explotación de hidrocarburos no convencionales, fracking, en las tres primeras provincias mencionadas, ya que en La Pampa no se han registrado debates y conflictos socioambientales respecto a esta actividad.

En Argentina, los estados provinciales tienen el dominio de los recursos naturales, por lo cual cada provincia posee legislaciones específicas para la regulación de las actividades que generan impactos ambientales, que se adicionan a las regulaciones de carácter nacional. Este factor es importante en materia ambiental, porque son las provincias las que otorgan los permisos ambientales para la explotación de recursos naturales, mediante la evaluación de los impactos ambientales declarados por las empresas proponentes de los proyectos. Es decir, si bien en Argentina existen leyes nacionales de "presupuestos mínimos" respecto a glaciares, agua, política ambiental y gestión de residuos, entre otras, estas son la base a partir de la cual las provincias pueden aumentar la protección ambiental en sus territorios. Así, como los procedimientos de Evaluación de Impacto Ambiental (EIA) se realizan a escala provincial, estos territorios se convierten en escenarios de debates y conflictos específicos. En este sentido, para los sectores sociales que resisten la instalación de proyectos extractivos, el gobierno provincial es un actor clave hacia el cual dirigir sus demandas.

La formación Vaca Muerta posee un área aproximada de $36.000 \mathrm{~km}^{2}$. Esta formación es parte de la cuenca neuquina (ver Figura 1$),{ }^{1}$ una de las mayores productoras de hidrocarburos en la porción austral de América del Sur, que cubre un área de más de $120.000 \mathrm{~km}^{2}$ en el centro-oeste argentino, siendo la cuenca hidrocarburífera con mayor actividad en Argentina (42\% en la producción de petróleo y 55\% en la de gas)

1 En la Figura 1 se decidió mapear la cuenca neuquina porque la formación geológica Vaca Muerta integra esta cuenca, pero se encuentra a diferentes profundidades y con diferentes grados de avance en su exploración. 

LUCRECIA WAGNER

(Delgado, 2018). Es una formación de tipo shale, lo cual significa que el petróleo y el gas se encuentran en una formación rocosa de baja porosidad y escasa permeabilidad. Para explotar estos recursos se utilizan métodos no convencionales de extracción, conocidos como fractura hidráulica o fracking. El fracking aumenta la permeabilidad de la roca madre para extraer los hidrocarburos por medio de la inyección de agua a alta presión en pozos, conjuntamente con la aplicación de agentes de sostén (arenas especiales y otros aditivos), lo que genera una fracturación de la roca y permite que los hidrocarburos atrapados en la formación se fluidicen hacia la superficie. La principal explotación se desarrolla en la provincia de Neuquén, en torno a la localidad de Añelo, pero también se utiliza este tipo de técnicas en las provincias de Río Negro y Mendoza. En las tres provincias, las actividades de fracking contaron con una importante promoción por parte del gobierno nacional ${ }^{2}$ y los gobiernos provinciales, aunque con procesos diversos de protesta social e insuficientes controles ambientales.

La provincia de Mendoza está situada en el centro-oeste de Argentina. Cabe decir que en esta provincia el uso del agua por parte de las élites configuró la organización espacial y social (Montaña, 2007; Martín, Rojas y Saldi, 2010; Prieto et al., 2020): la construcción de regadíos ha creado tres oasis que cubren alrededor del $4 \%$ de la provincia y albergan a más del 95\% de su población. La escasez de recursos en las zonas áridas hace de estos territorios un incuestionable campo de contiendas y la capacidad de imponer una decisión (en este caso, quién usa el agua, en dónde, de qué manera y para qué) constituye una manera de expresar el poder (Montaña, 2008).

La llegada del fracking a esta provincia, en un contexto de mega-sequía, movilizó a comunidades locales y sectores científico-académicos que alertaron sobre las consecuencias de iniciar este tipo de actividades sin considerar los riesgos ambientales que podría implicar, especialmente sobre el agua. En esta línea, el artículo busca mostrar cómo la inmediatez con la que el gobierno decidió autorizar el inicio de la explotación hidrocarburífera con técnicas de fracking en Mendoza, aumentó la incertidumbre relativa a esta actividad, ya que no se pudieron realizar los estudios necesarios y no se consideraron las diferentes opiniones y conocimientos sobre el desarrollo de esta técnica, ni la percepción de los riesgos por distintos actores de la sociedad.

A nivel metodológico, la investigación se sustenta en el trabajo de campo realizado en Mendoza durante el año 2018 (en Mendoza los debates y conflictos en torno al fracking se iniciaron posteriormente a los de Neuquén y Río Negro) y en la revisión de fuentes documentales. El trabajo de campo se basó en observación participante en espacios de debate sobre el fracking organizados por sectores académicos, legisladores mendocinos y organizaciones socioambientales. Estos eventos se realizaron principalmente en la Universidad Nacional de Cuyo y en la Legislatura de la Provincia de Mendoza. En estas instancias se procedió al registro de las principales demandas en materia ambiental y social expresadas por diferentes actores en estos debates. Sobre esa base se identificaron los ejes de discusión y las principales acciones del gobierno provincial que eran foco de críticas por parte de otros actores, haciendo hincapié en aquellas vinculadas a los procedimientos de impacto ambiental y a la participación social en instancias formales, por ejemplo, audiencias públicas. Estos ejes fueron

2 Al cierre de este trabajo, Vaca Muerta es presentada por el gobierno argentino como "un recurso de clase mundial que está cambiando la realidad energética del país" (Ministerio de Economía, s/f). 
Fracking en el sur de Mendoza: riesgos...

LUCRECIA WAGNER

analizados en relación con los ya identificados en la bibliografía existente sobre las problemáticas y consecuencias generadas por la explotación de hidrocarburos no convencionales, principalmente en Argentina, pero también considerando bibliografía que aborda diversos casos a escala global.

Por otra parte, se realizó una revisión documental de los materiales producidos por estos actores, así como también documentos oficiales sobre impactos ambientales de las actividades de fracking, legislación ambiental y otros informes oficiales y empresariales. El trabajo aquí expresado se basó en el análisis de estos documentos y de notas de campo de la autora.

Por último, estas instancias de trabajo se complementaron con la asistencia a protestas en el espacio público realizadas en la ciudad de Mendoza en rechazo al inicio del fracking en la provincia, especialmente las movilizaciones sociales en el centro de la ciudad de Mendoza entre abril y mayo de 2018, momento de mayor intensidad del conflicto en el espacio público. Además, se realizó un relevamiento de noticias sobre el tema durante 2018 y 2019 en medios de prensa provinciales, especialmente en los medios de prensa Los Andes y MDZ.

\section{La matriz energética argentina y su dependencia de los combustibles fósiles}

La matriz energética argentina está basada en los combustibles fósiles. Principalmente el petróleo y el gas proporcionan más del 85\% de la energía y, junto con las fuentes hidráulicas y nucleares, constituyen el $94 \%$ de esta matriz. Como estos datos sugieren, el país tiene una matriz energética poco diversificada y, en consecuencia, una alta concentración y dependencia de pocos recursos naturales (Wagner, Maurtua Konstantinidis y Blanco, 2018). Asimismo, el desarrollo de estos combustibles ha generado fuertes identidades sociales en algunas regiones del país, especialmente relacionadas con la empresa Yacimientos Petrolíferos Fiscales (YPF) (Muñiz Terra, 2007; Capogrossi, 2013), lo cual reduce las posibilidades de discutir sus impactos negativos. Ciertas comunidades tienen un fuerte arraigo con YPF y han naturalizado y/o aceptado los riesgos, o bien éstos son invisibilizados/minimizados por las empresas del sector y los gobiernos que reciben beneficios de estas explotaciones.

La llegada del fracking fue presentada por el gobierno nacional de la entonces presidenta Cristina Fernández de Kirchner como una gran oportunidad para recibir inversiones externas y alcanzar la soberanía energética nacional (Freier y Schaj, 2016). Algo que, con ciertas variantes, ha sido continuado por los gobiernos que la sucedieron, de Mauricio Macri y Alberto Fernández. De esta forma, los gobiernos nacionales argentinos han evitado el debate sobre los riesgos que esta actividad puede generar en materia ambiental, derivando la discusión hacia las oportunidades económicas que representa para el país la explotación del yacimiento Vaca Muerta.

En junio de 2010 se inaugura oficialmente la etapa de los hidrocarburos no convencionales, a partir de la perforación realizada por la empresa YPF-Repsol del primer pozo de shale en Loma de la Lata. Ya en 2011 la explotación de shale oil por parte de YPF había alcanzado los 5.000 barriles por día (Freier y Schaj, 2016). En este contexto 
Fracking en el sur de Mendoza: riesgos...

de creciente explotación de hidrocarburos no convencionales, la principal empresa dedicada a la explotación de petróleo y gas, YPF, fue parcialmente estatizada en 2012. La presencia de esta empresa fue un fuerte sostén para el argumento de alcanzar la "soberanía energética", aunque en la explotación de Vaca Muerta también participan empresas extranjeras como Chevron, muy cuestionada por su oscura historia de generación de impactos sociales y ambientales sobre las comunidades aledañas a sus sitios de explotación (Serrano Narváez, 2013; Pellegrini et al., 2020).

Si bien Argentina es un país con una importante organización de movimientos socioambientales que han tenido resultados exitosos como, por ejemplo, evitar la explotación minera en gran parte del país (Wagner y Walter, 2020) o de ciertas industrias y otros proyectos extractivos (Chiappe, 2005; Gutiérrez e Isuani, 2014), en el caso de la explotación de petróleo y gas el éxito de las demandas ambientales ha sido menor. El uso de la técnica de fracking ha generado cierta resistencia social, con diferentes ciclos de protesta y características propias en cada territorio provincial, pero esto no ha evitado la continuidad y crecimiento de la actividad.

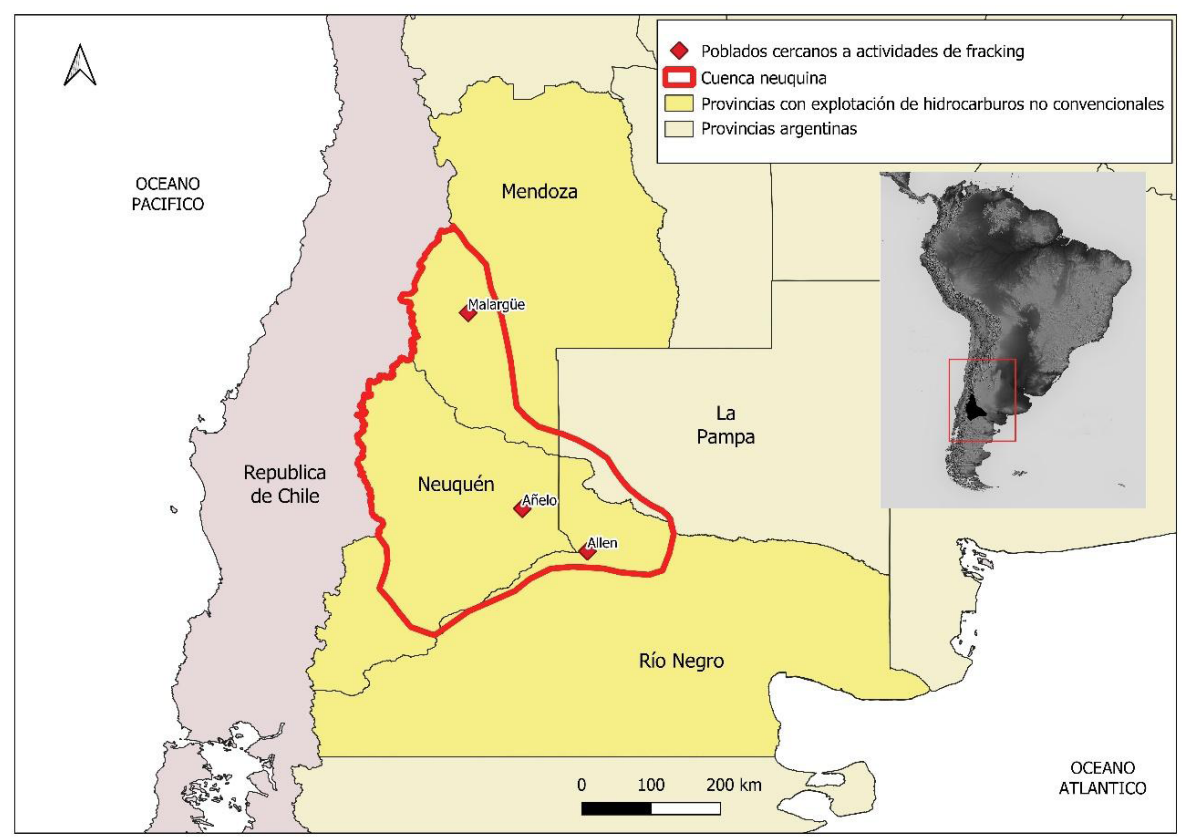

Figura 1. Territorios con explotación de hidrocarburos no convencionales en Argentina. Fuente: Facundo Rojas, basado en el Instituto Geográfico Nacional (IGN), Ministerio de Defensa, Presidencia de la Nación (2020); Sales et al. (2014); U.S. Geological Survey (2003).

\section{Fracking en Argentina: denuncias sobre la falta de consideración de riesgos e incertidumbre}

Uno de los primeros libros sobre las controversias del fracking en Argentina fue publicado en 2014, con el nombre de 20 Mitos y Realidades del Fracking (Bertinat et al., 2014). Los autores de este libro destacaron que, ante la falta de discusión en torno al fracking, se hacía necesaria la producción de un conocimiento experto independiente. De allí que decidieron producir y sistematizar información y conocimiento sobre esta técnica para contribuir a su difusión e impulsar la apertura de un debate democrático 
LUCRECIA WAGNER

en la sociedad argentina. El libro intentó romper la visión monolítica del fracking impulsada por el denominado "consenso fósil" imperante en Argentina. Un término que refiere a la existencia, durante las últimas décadas, de una política del sector dominada por los intereses corporativos que consolidaron una total dependencia hacia los hidrocarburos, tanto para el sistema energético en general, como para las provincias productoras en particular (OPSur, 2020). Un consenso que fue continuado por el "consenso del fracking" que reafirma la dependencia de los combustibles fósiles, pero también la dependencia de las grandes empresas trasnacionales, y no permite las disidencias ni el pensamiento crítico: "No solo clausura el camino para pensar en otras matrices energéticas, basadas en las energías limpias y renovables, sino también obtura el doble camino del debate societal y el de la democratización de las decisiones" (Svampa y Viale, 2014:351). Sin embargo, y a pesar de este consenso fósil, existen resistencias al avance hidrocarburífero y sus impactos en diferentes territorios del país, atravesadas también por los debates en torno a la transición energética y a modelos de desarrollo posibles.

Las advertencias sobre los riesgos e incertidumbres causados por la dinámica industrial y la opción por un crecimiento económico exponencial e ilimitado, durante décadas desestimadas en nuestro país, actualmente ocupan un lugar en la agenda de amplios movimientos sociales y políticos (Bertinat et al., 2014:9).

Como subraya Lorena Riffo (2017), desde distintos frentes de la resistencia social al fracking en Neuquén ha surgido la idea de que, más allá de una gestión más transparente y colectiva de la extracción, es necesario democratizar y des-comodificar los recursos energéticos, y buscar alternativas energéticas. Por su parte, Juan Carlos Radovich (2017) ha subrayado la necesidad de poner en práctica el acuerdo de Naciones Unidas sobre el Protocolo de Consulta Previa, Libre e Informada que reclaman las organizaciones indígenas, y promover mecanismos de control efectivos, en los que la participación popular debe estar garantizada por procedimientos democráticos. En este sentido, es importante destacar que los proyectos de fracking, principalmente pero no únicamente en Neuquén, han tenido graves conflictos con las comunidades Mapuche.

Revisando la literatura internacional en torno a la problemática del fracking se advierte que la escala local ocupa un lugar clave en la preocupación por los riesgos que puede acarrear esta técnica. Si bien el uso de la fracturación hidráulica es impulsado a nivel nacional, existe a nivel local una aceptación variable, encontrando comunidades en las que la tecnología y la infraestructura implementadas han sido permitidas habilitando las actividades de perforación, mientras que en otras localidades su implementación ha sido rechazada a partir de preocupaciones ambientales, dando lugar a regulaciones locales que limitan el fracking (Freier y Schaj, 2016).

En el caso argentino, las prohibiciones de fracking se han gestado a nivel local vía sanción de ordenanzas emitidas por los poderes legislativos municipales, ${ }^{3}$ y a nivel provincial para el caso de la provincia de Entre Ríos. Estas prohibiciones están a menudo

3 Numerosas organizaciones han impulsado medidas a nivel local que, en el plano institucional, se tradujeron en la aprobación de ordenanzas que prohibieron la aplicación del fracking en municipios. En 2014 se contaban más de 30 ordenanzas en las provincias de Río Negro, Neuquén, Chubut, Buenos Aires, Mendoza y Entre Ríos (Bertinat et al., 2014:43). Se recomienda consultar el cuadro de la página 177 del libro de Bertinat et al. 2014 que detalla los municipios que sancionaron ordenanzas. Otras leyes y ordenanzas fueron sancionadas posteriormente, como la ley provincial que prohíbe el fracking en Entre Ríos (Ley N¹0.477/2017). Al 2017 existían más de 50 ordenanzas que prohíben el fracking en Argentina (Aranda, 2017). 
Fracking en el sur de Mendoza: riesgos...

acompañadas de un alto grado de incertidumbre pública sobre los daños locales (Boudet et al., 2014; Hess et al., 2019). La discusión sobre el riesgo ambiental local y la contaminación dominan el discurso contra el fracking en toda Europa, Argentina y Estados Unidos (Williams y Sovacool, 2019). En Argentina, los tres niveles gubernamentales -local, provincial y nacional-, que comparten la responsabilidad de supervisar y regular el gas de esquisto, no han abordado adecuadamente la preocupación local por el riesgo (Saulino, 2018). Si bien a nivel municipal se han sancionado ordenanzas prohibitivas, la preocupación por el riesgo excede estas instancias institucionales, que además están sujetas a la posibilidad de ser declaradas inconstitucionales por los tribunales superiores de justicia a escala provincial, como ha sucedido en Allen, Río Negro, en 2013. ${ }^{4}$

Las externalidades sociales y ambientales de la explotación de hidrocarburos no convencionales en Vaca Muerta han sido analizadas por Martín Álvarez Mullally et al. (2017). El objetivo del informe realizado por estos autores fue "abrir la discusión sobre los riesgos y las incertidumbres de los impactos cuestionando el 'conocimiento experto', el 'discurso oficial' y las limitaciones del sistema normativo y de los organismos de control y peritaje" (Álvarez Mullally et al., 2017:15). A su vez, destacan la existencia de diferentes percepciones del riesgo en cada sector social: "El contraste de las heterogéneas percepciones contribuye a entender mejor el fenómeno que los números fríos de un especialista que no necesariamente gozan de consenso social" (Álvarez Mulally et al., 2017:33).

Un tema clave en torno al cual existe un alto nivel de incertidumbre es la actividad sísmica percibida por los habitantes de poblados cercanos a áreas en las que se utilizan las técnicas de fracking en Neuquén. Los expertos han subrayado que, al no haber mediciones previas, una red de monitoreo permanente debería ser indispensable para generar conocimiento sobre las causas de la actividad sísmica en Vaca Muerta (Trifogli, 2020). Además, existen algunas investigaciones que muestran relación entre el aumento de la actividad sísmica y las zonas donde se desarrolla la explotación mediante fracking (Calalesina, 2021).

Otra cuestión clave que genera preocupación sobre la sostenibilidad de las actividades de fracking es su demanda de cantidades significativas de energía, agua y materiales, incluyendo acero, cemento y arena (Ferrante y Giuliani, 2014). Estos autores afirman que, si la actividad se expande según las perspectivas analizadas en su investigación, puede implicar transformaciones en la escala del cambio territorial de una magnitud aún no prevista. En cuanto al agua, existe un conocimiento limitado de los impactos que su extracción podría tener sobre los recursos hídricos locales (Rosa y D’Odorico, 2019).

Por otra parte, Juan Acacio y Fernando Svampa (2017) subrayan que los EIA no destacan el carácter experimental de esta técnica, ni la falibilidad humana que puede generar desastres ambientales y afectar al suelo, las aguas subterráneas, el aire y la salud pública. Tampoco consideran los múltiples desplazamientos de otras actividades económicas, la expulsión de comunidades de sus tierras ancestrales generada por la actividad hidrocarburífera (ver Hadad, Palmisano y Wahren, 2020), los impactos urbanos en el paisaje local, y mucho menos los debates sobre el uso del agua y el suelo o los vertederos generados.

4 El Concejo Deliberante de la ciudad de Allen, Río Negro, había sancionado en agosto de 2013 una ordenanza que la declaraba "libre de fracking" (Ordenanza N046/2013) impulsada por asambleas y organizaciones locales. Tres meses después, el Superior Tribunal de Justicia de la provincia de Río Negro declaró inconstitucional esta ordenanza a través de la Sentencia N¹35 D. Más información en: Río Negro (2013). 
LUCRECIA WAGNER

En reacción a ello, las organizaciones sociales que resisten a las actividades de fracking han construido un corpus de información relevante respecto a esta técnica. Los impactos ligados a la actividad tuvieron varios focos de conflicto que provocaron diferentes niveles de organización y movilización (Wyczykier y Acacio, 2021). Dichas organizaciones se convirtieron en actores clave en el proceso de definición del fracking como problema público. Estos grupos realizaron diagnósticos sobre los impactos de la actividad hidrocarburífera desde perspectivas económicas, sociales, políticas y culturales (Acacio y Wyczykier, 2020).

\section{Perspectivas teóricas: incertidumbre, análisis de riesgos y participación social}

En este trabajo argumentamos que las evaluaciones ambientales de las explotaciones de hidrocarburos no convencionales y las oportunidades de participación social fueron menos rigurosas con el fin de facilitar el inicio de las técnicas vinculadas al fracking en la provincia de Mendoza. Este argumento se basa, principalmente, en los siguientes hechos. Por un lado, la decisión de la autoridad ambiental provincial de no realizar una evaluación de impacto ambiental específica para la actividad y, en lugar de ello, llevar adelante una evaluación básica y sin audiencia pública. Por otro, la falta de consideración de las recomendaciones hechas por el dictamen técnico, ${ }^{5}$ que destacó la ausencia de determinados estudios y la necesidad de contar con esta información, además de recomendar la realización de ciertos controles, por ejemplo, mediante la instalación de freatímetros antes del inicio de la actividad. ${ }^{6}$

Nuestra hipótesis es que la incertidumbre asociada a los impactos ambientales del fracking fue incrementada por el accionar de gobiernos y empresas al avanzar con la actividad sin estudios ambientales más rigurosos, a lo que se suma la falta de consulta a la población bajo riesgo, tanto local como provincial.

La importancia de considerar la incertidumbre en el conocimiento científico ha sido explorada por Funtowicz y Ravetz (1993), utilizando el término ciencia posnormal. Este tipo de ciencia es necesaria cuando los hechos son inciertos, los valores están en disputa, hay mucho en juego y las decisiones son urgentes. Los autores explican que, en estos casos, la garantía de calidad de las aportaciones científicas al proceso político requiere una "comunidad de pares extendida”, formada por todos quienes tienen un interés en dialogar sobre el tema (Funtowicz y Ravetz, 1993:739). Uno de los aspectos importantes que puede aportar la ciencia posnormal es una vía para la democratización de la ciencia (Funtowicz y Ravetz, 1993).

5 Dictamen Técnico del Informe de Adecuación de Infraestructura relativo al proyecto Estimulación Hidráulica en los Pozos Identificados como PETRE Md.N. CP-1013, PETRE Md.N. CP-1015, PETRE Md.N. CP-1017 e YPF Md. Nq. PR-59, Área de Concesión Puesto Rojas, departamento de Malargüe, provincia de Mendoza. Realizado por la Fundación CRICYT el 26 de julio de 2017.

6 Algunos ejemplos de la falta de información detallados en el Informe Técnico de la Fundación CRICYT son: no se especifican lugar ni condiciones de almacenado de los productos químicos; la descripción de la geología es regional y a una escala que no es útil para determinar los impactos a causa de la estimulación; en un estudio ambiental, la geomorfología es una herramienta para predecir-prevenir posibles riesgos geológicos (inundaciones, aludes, flujos de barro, derrumbes, cárcavas, colapsos por disolución, etc.), dado que estos no pueden ponderarse con una descripción a escala regional, es necesario un relevamiento local; no se presentan los caudales medios, mínimos ni máximos del río Salado, un dato importante considerando que se tomará agua del río. A su vez, el informe recomienda: desarrollar un mapa de sensibilidad ambiental para el Puesto Rojas y Cerro Pencal elaborado por especialistas; en virtud del potencial riesgo a las capas subsuperficiales, se sugiere monitorear (antes y después de cada intervención) la calidad del recurso subterráneo mediante la instalación de freatímetros aguas abajo del sitio de las operaciones. 
Es decir que la gestión de la incertidumbre se basa en la ampliación de la participación en la discusión y toma de decisiones. Basándose en un estudio sobre Polonia, Aleksandra Lis y Agata Stasik (2017) han destacado que la situación de incertidumbre es también una situación de déficit de conocimiento compartido que se distribuye, aunque de forma desigual, entre los expertos, la administración y el público lego. Los déficits de conocimiento pueden empujar a los actores a producir un conocimiento que les otorgue una visión más "certera" del futuro y una posibilidad de actuar en consecuencia (Lis y Stasik, 2017:35). Pero si además de la falta de conocimiento no se impulsa la participación social y, por el contrario, como se muestra a continuación, se la limita criminalizando las voces críticas, el contexto de incertidumbre se amplifica a costa de la posibilidad de evaluar los riesgos ambientales que son, justamente, los que permiten anticipar lo que puede pasar y obrar en consecuencia para disminuir o evitar impactos negativos.

Las disputas por el conocimiento en los conflictos ambientales han sido denominadas controversias sociotécnicas (Callon, Lascoumes y Barthe, 2001; Merlinsky, 2013), mientras que el conocimiento generado por los movimientos sociales, y los académicos y científicos que los apoyan, como contra-experto o contra-hegemónico (Svampa y Antonelli, 2009).

En Argentina, el conocimiento científico ha jugado un papel relevante en las disputas ambientales, así como la producción de diversos tipos de conocimiento que han sido desplegados en los debates sobre ciertas actividades extractivas (Svampa y Antonelli, 2009; Arancibia y Motta, 2018). En el caso de la megaminería, sectores científicos y académicos han colaborado con movimientos socioambientales generando y sistematizando conocimiento sobre los impactos ambientales y sociales de esta actividad. Esto fue clave para contrarrestar la información técnica y las evaluaciones de impacto ambiental generadas por las empresas mineras y las consultoras ambientales contratadas por estas empresas (Wagner y Walter, 2020). Mendoza es un caso emblemático en este aspecto, en el cual las asambleas y otras organizaciones socioambientales participaron de los procedimientos de evaluación de impacto ambiental de los dos proyectos que llegaron a evaluación ambiental de la etapa de explotación: Potasio Río Colorado y San Jorge (Wagner y Giraud, 2011; 2013). Aun cuando ambos proyectos fueron aprobados en estas instancias, en el caso del proyecto San Jorge, las críticas y la oposición social para su instalación fueron claves para que la legislatura de Mendoza rechazara el proyecto en 2011. En estos casos, a diferencia de lo acontecido posteriormente respecto al fracking, las voces disidentes fueron escuchadas y el Estado provincial generó espacios de debate, siguió los procedimientos de evaluación de impacto ambiental determinados por la legislación ambiental -incluyendo audiencias públicas- y amplió los espacios de discusión, incluso en el marco de la legislatura provincial.

Es decir que las disputas en torno a la megaminería en Argentina, dentro de las cuales Mendoza es un caso emblemático, constituyen un ejemplo de cómo los movimientos socioambientales, comunidad científica y académica y otros actores sociales reaccionaron rápidamente contrastando datos e información aportados por las empresas y gobiernos. A diferencia de lo que aconteció con el fracking, cuya falta de debate público dificultó este tipo de experiencias.

Otro caso relevante en Argentina ha sido el de la contaminación por pesticidas, en el que la información necesaria para poder regular esta actividad no fue generada a tiempo, 
proceso similar al que actualmente ocurre con el fracking. Esto lleva al problema de la undone science (ciencia no hecha), es decir, el conocimiento que sistemáticamente no se produce y que sería útil para un movimiento social u organización de la sociedad civil que trabaje para promover el cambio y/o resistir las políticas que consideran perjudiciales (Arancibia y Motta, 2018). A ello se sumó la persecución ideológica y académica denunciada por equipos de investigación argentinos que realizaron investigaciones y presentaciones públicas de las mismas, problematizando los efectos de los agrotóxicos sobre comunidades humanas (Aranda, 2014; Página12, 2016).

El derecho de las comunidades locales a decidir sobre las actividades que podrían desarrollarse en sus territorios aparece en la bibliografía como un tema clave en las disputas en torno a la explotación de recursos naturales. Respecto al fracking, en un artículo basado en casos de diferentes países, se muestra cómo las autoridades locales denegaron inicialmente el permiso para el fracking y luego las decisiones para aprobar la actividad fueron tomadas a nivel nacional (Aczel, Makuch y Chibane, 2018). Otra acción polémica desarrollada por los gobiernos es la criminalización de las protestas contra el fracking. Estas protestas están interrelacionadas con otras demandas, como las que pujan por frenar el cambio climático y avanzar hacia una transición energética. Paradójicamente, como ha destacado Timothy O’Riordan (2015), lo que resulta aún más insidioso es la vigilancia sistemática de las protestas contra el cambio climático en una democracia en la que la comunidad científica de todas las disciplinas reclama precisamente esa movilización.

Natenzon (2015) fue impulsora, a mediados de la década de 1990, de un análisis del riesgo con aplicación práctica, que consideraba cuatro componentes: peligrosidad y vulnerabilidad social, que ya eran aceptadas en la literatura sobre el tema, a las cuales proponía agregar exposición e incertidumbre. Mientras que la peligrosidad era relativa a eventos causales, naturales o antrópicos, la incertidumbre era considerada un aspecto clave vinculado a los valores en juego, la toma de decisiones y el poder.

Posteriormente, la autora, junto a otros investigadores, fueron desarrollando cambios en esta propuesta inicial. Por ejemplo, se jerarquizaron las componentes, entendiendo que exposición e incertidumbre son las más complejas y que surgen como resultado de combinaciones de peligrosidad y vulnerabilidad, que serían componentes más simples. Además, la exposición se identificó como componente independiente, en cuanto expresión territorial de combinaciones históricas de peligrosidades y vulnerabilidades sociales. Natenzon destacó que el diagnóstico de la exposición puede dificultarse debido a la ausencia o fragmentación del conocimiento sobre el territorio, la falta de participación de quienes corren el riesgo en la reconfiguración de ciertas áreas, la toma de decisiones en función de intereses rentísticos o las falencias de los organismos de planificación y control:

Puede decirse que la incertidumbre es el inverso del riesgo. Es un error decir que no hay riesgo cuando algo es seguro. En realidad, algo es seguro cuando se puede neutralizar la peligrosidad, que, es solo una componente del riesgo. Cuando no se pueden caracterizar las tres dimensiones (peligrosidad, vulnerabilidad y exposición), cuando no hay conocimiento sobre ellas, ya no se trata de "riesgo" sino de "incertidumbre" (Natenzon, 2015:XVIII). 
Como destaca esta autora, cuando el riesgo es no cuantificable se transforma en incertidumbre (ver Figura 2). Cuestión que resulta clave para analizar la problemática que aquí abordamos: las zonas grises del conocimiento científico ponen de manifiesto el carácter político-valorativo de las decisiones. Su aceptación dependerá de complejos mecanismos de legitimación entre el discurso científico y las prácticas políticas, y de cuan democrática sea la toma de decisiones.

La incertidumbre ha ido adquiriendo importancia para la gestión del riesgo. El carácter más o menos peligroso de un evento está asignado socialmente. Es decir, la cualidad peligrosa de los fenómenos o procesos está mediada socialmente por un sujeto que las define como tal. Además, puede aparecer una "peligrosidad amplificada" por la imprevisión técnica, institucional y política a lo largo del tiempo. En cuanto a la vulnerabilidad social,

(...) compleja y multidimensional, abarca aspectos tales como las condiciones de vida de la población, los marcos normativos e institucionales que regulan su accionar, así como las carencias y limitaciones en la toma de decisiones. La vulnerabilidad social es, probablemente, la dimensión menos explorada del riesgo (Natenzon, 2015:XV).

Natenzon destaca aspectos materiales y simbólicos que conforman la vulnerabilidad, entre los que se encuentran las instituciones, la organización social, la forma en que se ejerce la legalidad, los caminos para resolver conflictos y, en definitiva, el contexto democrático en el que se desarrolla la vida cotidiana.

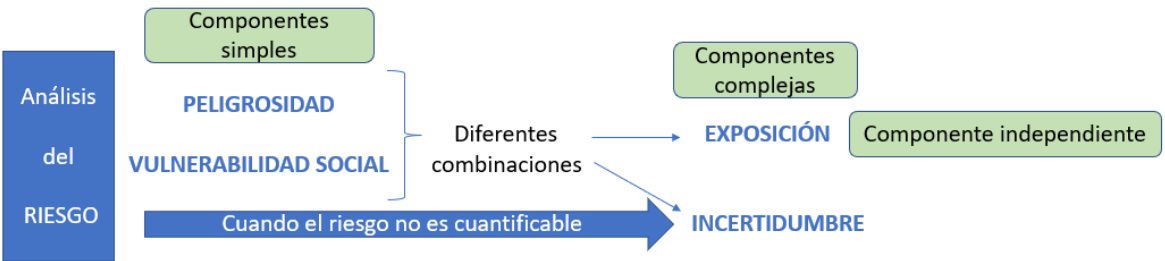

Figura 2. Componentes del análisis de riesgo. Fuente: elaboración propia en base a Natenzon (2015).

\section{Riesgo, incertidumbre y resistencias en torno al fracking en Mendoza}

Mendoza es una provincia árida, que sufre desde hace más de diez años una intensa sequía. En diciembre de 2019, ante la intención del gobierno provincial de modificar la Ley $\mathrm{N}^{\circ} 7722$ que restringe la minería metalífera, con el fin de avanzar con esta actividad en la provincia, investigadores del Instituto Argentino de Nivología, Glaciología y Ciencias Ambientales (IANIGLA) emitieron un comunicado que expresaba:

Los mendocinos no estamos exentos de los desafíos que impone la crisis ambiental planetaria. Por la falta de nieve en la Cordillera, Mendoza vive la sequía más extendida de los últimos 110 años. Desde el invierno del año 2010, nuestra provincia se encuentra en emergencia hídrica. (...) Un elemento central a 
considerar, sobre todo en la parte alta de nuestras cuencas hídricas, son los riesgos potenciales de las actividades que se propone autorizar. ¿̇s necesario autorizar actividades extractivas de alto riesgo e impacto ambiental en ambientes donde se genera el agua que consumimos los mendocinos? (Unidiversidad, 2019).

En Mendoza, el fracking comenzó en 2017 en el sur de la provincia, en la zona de Malargüe, sin los requisitos ambientales necesarios para disminuir el riesgo de esta actividad. Paradójicamente, Mendoza es una de las provincias argentinas donde el rechazo a la megaminería ha tenido más éxito. En una región árida, su "cultura de oasis" ha sido uno de los argumentos de oposición a esta actividad (Wagner, 2019). En esta línea, desde 2004 se organizó una fuerte red de vecinos autoconvocados, autodenominados Asambleas por el Agua, para rechazar los proyectos de megaminería; organización que también fue clave en las protestas contra el fracking.

Mendoza es la cuarta provincia en extracción de crudo y gas convencional del país. Su actividad hidrocarburífera contribuye con un 25\% al Producto Bruto Geográfico (PBG) provincial (Mendoza Invest, 2016). En el caso del fracking, para lograr avanzar rápidamente y neutralizar una oposición social que pudiera emular la resistencia a la minería a gran escala, el gobierno provincial presentó a esta actividad como la continuidad de explotaciones convencionales existentes. Este argumento también fue utilizado para evadir la necesidad de presentar una evaluación de impacto ambiental de la explotación mediante fracking. Así, el fracking se inició sin audiencia pública, en contradicción con la legislación ambiental nacional y provincial. Las primeras fracturas hidráulicas en shale fueron hechas por YPF en 2012, en dos pozos del departamento Malargüe, con una evaluación ambiental que no se diferenciaba de las aplicadas en las explotaciones convencionales y sin divulgación social. Algunos departamentos mendocinos acompañaron las luchas contra el fracking en otras provincias y sancionaron legislación restrictiva a escala municipal (Gutiérrez Ríos, Wagner y Giraud, 2021). En 2017, se realizó una prueba piloto de estimulación hidráulica en pozos petroleros preexistentes en Cerro Pencal-Puesto Rojas, a $20 \mathrm{~km}$ de la ciudad de Malargüe, autorizada por el gobierno provincial. Allí se conoció públicamente la llegada del fracking a Mendoza.

La aplicación de legislación ambiental para regular esta actividad fue muy laxa. Como no existía en la provincia una normativa particular para el fracking, esta técnica hubiera requerido una manifestación de impacto ambiental, tal como fue sugerido por el Área de Petróleo que funciona dentro de la Dirección de Protección Ambiental (DPA), la autoridad ambiental del gobierno provincial:

El Área de Petróleo, dentro de la DPA, generó un informe técnico que señalaba que, a falta de legislación sobre esta técnica en especial, debía solicitarse una manifestación de impacto ambiental específica. Apartándose de la opinión técnica, la directora de la DPA consideró que, datando la concesión de los años 70 y que PETSA como concesionaria desde 2006 había dado cumplimiento al Decreto 170/2008 (artículo 9), la presentación e información brindada por la empresa era suficiente para evaluar los impactos ambientales (Nievas, 2019:214).

7 Este decreto en su Artículo $N^{\circ} 9$ determina que las empresas de exploración y explotación de hidrocarburos que desarrollaban actividades en la provincia de Mendoza debían presentar un Informe Ambiental 2008 a modo de evaluación ambiental de la actividad y detalla los contenidos del mismo. 
Fracking en el sur de Mendoza: riesgos... LUCRECIA WAGNER

Así, el gobierno provincial, a través de su DPA autorizó la presentación de un proceso de evaluación ambiental abreviado y sin audiencia pública, denominado Aviso de Proyecto ${ }^{8}$ por "readecuación de infraestructura". Como destaca Nievas (2019), resoluciones como la N $589 / 2014$ de Mendoza, que regulan cómo instrumentar la adecuación de infraestructura para obras no contempladas, crean procedimientos administrativos para la evaluación rápida de obras pequeñas que deben ser resueltas en poco tiempo por razones de seguridad o debido a su escasa magnitud. Pero, aplicadas a actividades como el uso de técnicas de fracking, implican "un grave retroceso en la eficacia del Derecho Ambiental: se reducen, contra la letra y el espíritu de la Ley $5.961,{ }^{9}$ los casos en que debe realizarse evaluación de impacto ambiental -la regla- 0 aviso de proyecto -procedimiento reducido en casos excepcionales. Sobre estas bases se aprobó la entrada del fracking en Mendoza” (Nievas, 2019:214).

La Fundación del Centro Regional de Investigaciones Científicas y Tecnológicas (CRICYT), perteneciente al Consejo Nacional de Investigaciones Científicas y Técnicas (CONICET), actuó como ente dictaminador técnico, al tiempo que el Departamento General de Irrigación (DGI) y el Municipio de Malargüe elaboraron informes sectoriales. ${ }^{10}$ Este procedimiento de evaluación fue aprobado por la Resolución $\mathrm{N}^{\circ} 813 / 17$ de la Dirección de Protección Ambiental, que no tuvo en cuenta numerosas recomendaciones de estos organismos dictaminadores.

Por ejemplo, se realizó fracking en pozos sin haber instalado previamente freatímetros para establecer una línea de base de calidad del agua subterránea, y no se realizaron estudios más exhaustivos sobre componentes del ecosistema y sismicidad que habían sido recomendados por el dictamen técnico de la Fundación CRICYT. Ante ello, organizaciones ambientales denunciaron penalmente a los responsables del área ambiental de la provincia, causa judicial que ha logrado escaso avance, pero sigue abierta (Gutiérrez Ríos, Wagner y Giraud, 2021).

Posteriormente, y gracias a las demandas sociales que reclamaban una audiencia pública, el gobierno provincial finalmente elaboró un decreto para regular la actividad del fracking (Decreto $\mathrm{N}^{\circ} 248 / 2018$ ) y realizó la audiencia. Sin embargo, los movimientos socioambientales de la provincia no consideran que este decreto sea una herramienta suficiente para garantizar la seguridad de la actividad (Gutiérrez Ríos, Wagner y Giraud, 2021). Además, organizaciones nacionales demandaron su inconstitucionalidad, ya que no aborda exhaustivamente impactos relevantes de la actividad, no garantiza la falta de independencia de quienes realizan los estudios

8 La exploración y explotación de hidrocarburos están incluidas entre las actividades sometidas al proceso de evaluación de impacto ambiental por la autoridad ambiental provincial, según la Ley Provincial N⒌961, de Preservación del Medio Ambiente. El Decreto №2.109/94 establece la excepción para aquellos proyectos que por su escaso impacto o magnitud no puedan afectar el equilibrio ecológico de uno o más ecosistemas. "Se entenderá que las obras o actividades comprendidas en el proyecto puedan previsiblemente alterar el equilibrio ecológico, cuando éstas puedan superar la capacidad de carga del ecosistema" (Artículo 9). Para la obtención de esta exención, el proponente deberá presentar el Aviso de Proyecto.

9 Se trata de la Ley de Preservación del Medio Ambiente de la provincia de Mendoza.

10 Algunos detalles sobre el dictamen técnico de Fundación CRICYT fueron presentados en nota al pie $\mathrm{n}^{\circ} 6$. Asimismo, el DGI estableció que la empresa debía informar el inicio de los trabajos de estimulación hidráulica para programar inspecciones durante el desarrollo de los trabajos y realizar análisis fisicoquímicos de las aguas de retorno (flowback), entre otras recomendaciones. 
sobre estos impactos y cambia las prioridades en cuanto al uso del agua (destacan que se prioriza más el fracking que el agua para uso de los productores agrícolas). ${ }^{11}$

Por otra parte, las movilizaciones sociales fueron neutralizadas y controladas. Si bien en abril de 2018 se visibilizaron protestas contra el fracking en diferentes localidades de la provincia -masivas en el departamento de General Alvear-, el gobierno provincial reaccionó rápidamente contra las opiniones críticas, acusándolas de mentir y generar pánico en la población. El ejemplo más extremo de esta situación fue la denuncia penal por parte de diputados provinciales oficialistas hacia una persona que había enviado un audio de WhatsApp que se hizo viral, por "generar pánico en la población”, lo cual fue considerado por ciertos sectores como un ataque a la libertad de expresión (Perez, 2018). ${ }^{12}$ El propio gobernador de la provincia de Mendoza denunció la existencia de una "campaña de mentiras para meterle miedo a la ciudadanía" (Brusotti, 2018).

A su vez, las manifestaciones públicas fueron rápidamente intimidadas (Salomone, 2021). En abril de 2018, unos camiones con insumos que se dirigían a la explotación vía fracking en Malargüe fueron detenidos en San Carlos, población donde nació el rechazo contra la megaminería. Una masiva concentración de personas, principalmente alumnos y docentes de una escuela local, a quienes se sumaron vecinos que fueron llegando a la ruta, bloquearon la autopista durante más de nueve horas. Intervino la policía nacional y provincial, y algunos vecinos fueron imputados por un juez federal, una práctica de intimidación que no se había dado en la provincia de Mendoza ante situaciones similares, como las de rechazo a la megaminería.

\section{Reflexiones finales}

Este trabajo muestra cómo, en la provincia de Mendoza, en primer lugar, la incertidumbre es ampliada mediante la utilización de ciertas figuras existentes en el marco normativo ambiental, como "aviso de proyecto", para adaptarlo a la necesidad gubernamental y empresarial de acelerar la extracción de hidrocarburos no convencionales. Como consecuencia de estas acciones, el riesgo no puede ser analizado y, tal como destaca Natenzon (2015), cuando esto sucede pasamos al campo de la incertidumbre. Incertidumbre que es gestionada por los actores de poder al generar condiciones para la población expuesta, como el acallamiento de sus voces críticas mediante acciones coercitivas por parte del gobierno provincial y la negativa para comunicar la existencia de peligrosidades.

Así, si lo comparamos con los debates en torno a otras actividades cuestionadas, como la megaminería, el fracking constituye en Mendoza y en el país un caso de regresión democrática de los debates socioambientales. En consecuencia, es importante destacar que la falta de contexto democrático en el que se desarrolla la vida cotidiana es otro de los elementos que contribuye a la amplificación de la vulnerabilidad social, como puede verse a partir de las denuncias hacia las voces disidentes y la criminalización de la protesta.

11 Entre ellas, Fundación Ambiente y Recursos Naturales (FARN), Federación Argentina de Espeleología (FADE), Fundación Oikos Red Ambiental y Asociación Argentina de Abogados Ambientalistas (AAdeAA). Más detalles sobre estas demandas en Nievas (2019) y AAdeAA (2021).

12 Para mayor detalle sobre estos hechos se recomienda consultar la nota de Mariano Salomone (2019). 
Considerando las investigaciones existentes sobre la problemática socioambiental del fracking en Argentina, puede concluirse que existen preocupaciones y demandas por parte de comunidades cercanas a las explotaciones, organizaciones socioambientales, investigadores y académicos de diferentes disciplinas y población en general debido a la falta de información oficial otorgada por los gobiernos y las empresas respecto a las consecuencias de esta actividad. Los artículos ya existentes sobre la problemática generada por la explotación de hidrocarburos no convencionales mediante la técnica de fracking, que han servido de referencia para este trabajo, muestran y destacan la desconfianza de las comunidades locales en relación con la visión monolítica del fracking detentada por gobiernos y empresas, que sólo resaltan los beneficios económicos de la actividad. Varios estudios realizados sobre las provincias en las que existe debate sobre el fracking, donde se generan conflictos y controversias, muestran que los impactos sociales y ambientales no han sido considerados ni discutidos, y que las preocupaciones de las poblaciones locales no han sido tenidas en cuenta. De esta forma, la incertidumbre inherente a las actividades de fracking no sólo no está siendo abordada por los discursos oficiales, sino que está siendo incrementada al no abrirse el debate entre las percepciones y conocimientos de los distintos sectores sociales en torno a los riesgos e impactos de la actividad sobre las sociedades y los territorios. Ante ello, los científicos y académicos que han estudiado los conflictos e implicaciones territoriales del fracking, junto a las organizaciones socioambientales que resisten a la actividad, abogan por un conocimiento experto independiente y por la apertura de un debate democrático.

A su vez, la falta de generación del conocimiento necesario para la toma de decisiones dificulta el diagnóstico de la exposición a la cual la población mendocina está siendo sometida, en un contexto de mega-sequía, favoreciendo, por el contrario, la toma de decisiones en función de intereses empresariales y de sectores gubernamentales afines a las prácticas relacionadas al fracking. Esta falta de conocimiento también favorece el paso de un contexto de riesgo a un contexto de incertidumbre. Por ende, la problemática en torno al fracking es un caso que amerita la conformación de una "comunidad ampliada de pares", que debería incluir a todos aquellos con interés en discutir las implicaciones de esta actividad.

En definitiva, el avance basado en la falta de conocimiento e inconsulto del fracking en Mendoza es considerado un caso emblemático de amplificación de la incertidumbre. Sobre todo, tomando en cuenta que, en otros debates socioambientales relevantes a escala provincial y nacional, esta provincia fue un caso ejemplar en la apertura de espacios de debate e incluso de sanción de legislación y regulación sobre el tema, ampliando las comunidades de pares extendidas. En el caso de la megaminería, si bien ese debate fue impulsado por los movimientos socioambientales, el Estado abrió los espacios correspondientes para que pudiera canalizarse también por vías institucionales. Por el contrario, en el caso del fracking, los entes gubernamentales flexibilizaron las regulaciones, mientras que las reglamentaciones sancionadas son consideradas un retroceso para el derecho ambiental. Sumado a ello, las voces críticas fueron acalladas y duramente cuestionadas, con lo cual Mendoza constituye una geografía más donde el consenso fósil imperante se pone en práctica con su más cruda evidencia.

Desde esta conclusión, preocupa que la falta de consideración de las demandas socioambientales en torno a las técnicas de fracking vaya reduciendo la posibilidad de una discusión participativa mediante la cual pueda gestionarse la incertidumbre imperante. 
Fracking en el sur de Mendoza: riesgos...

LUCRECIA WAGNER

Quienes gestionan con conocimiento, gestionan el riesgo. Pero si la población involucrada territorialmente en las acciones a implementar no es informada ni consultada, el proceso se inscribe en un marco de incertidumbre. Además, será esta población la que reciba los impactos negativos que, habiéndose podido evitar, no fueron gestionados preventivamente.

La autora agradece al Consejo Nacional de Investigaciones Científicas y Técnicas (CONICET) y al Proyecto de Investigación Científica y Tecnológica Orientado (PICTO) financiado por la Agencia Nacional de Promoción Científica y Tecnológica y la Universidad Nacional de Cuyo (PICTO oo12-2016 UNCuyo), por el apoyo institucional y financiero para desarrollar esta investigación. A los coordinadores de este dossier y a los evaluadores de este artículo, cuyas sugerencias y correcciones permitieron mejorar sustancialmente este trabajo. 


\section{Q Bibliografía}

» AAdeAA (2021). Los caminos judiciales del fracking: activismo judicial estratégico como resistencia al avance ilegal del fracking y la explotación de hidrocarburos no convencionales. El caso de la provincia de Mendoza. Asociación Argentina de Abogados Ambientalistas.

» Acacio, J.A. y Svampa, F. (2017). Hidrocarburos no convencionales y fracking: Estado, empresas y tensiones territoriales en la Patagonia argentina. Cuestiones De Sociología, 17, e038. DOI: https://doi.org/10.24215/23468904e038

»Acacio, J.A. y Wyczykier, G. (2020). Expectativas públicas y conflictos sociales en torno a los hidrocarburos no convencionales en Argentina: algunos apuntes sobre Vaca Muerta. Izquierdas, 49, 457-477.

»Aczel, M.R., Makuch, K. E. y Chibane, M. (2018). How much is enough? Approaches to public participation in shale gas regulation across England, France, and Algeria. The Extractive Industries and Society, 5(4), 427-440. DOI: https://doi. org/10.1016/j.exis.2018.10.003

» Álvarez Mullally, M., Arelovich, L., Cabrera, F. y Di Risio, D. (2017). Megaproyecto Vaca Muerta. Informe de externalidades. Buenos Aires: EJES-Enlace por la Justicia Energética y Socioambiental.

" Arancibia, F. y Motta, R. (2018). Undone Science and Counter-Expertise: Fighting for Justice in an Argentine Community Contaminated by Pesticides. Science as Culture, 28(3), 277-302. DOI: https://doi.org/10.1080/09505431.2018.1533936

» Aranda, D. (2017, 08 de mayo). No habrá fracking en Entre Ríos. Página12. Recuperado de: https://www.pagina12.com.ar/36346-no-habra-fracking-en-entrerios

» Aranda, D. (2014, 13 de mayo). Andrés Carrasco: "Pocas cosas más peligrosas que la verdad". Territorios. Recuperado de: https://www.darioaranda.com. ar/2014/05/andres-carrasco-pocas-cosas-mas-peligrosas-que-la-verdad/

» Bertinat, P., D’Elia, E., Observatorio Petrolero Sur, Ochandio, R., Svampa, M. y Viale, E. (2014). 20 Mitos y Realidades del Fracking. Buenos Aires: Editorial El Colectivo.

» Boudet, H., Clark, C., Bugden, D., Maibach, E., Roser-Renouf, C. y Leiserowitz, A. (2014). "Fracking" controversy and communication: Using national survey data to understand public perceptions of hydraulic fracturing. Energy Policy, 65, 57-67. DOI: https://doi.org/10.1016/j.enpol.2013.10.017

» Brusotti, F. (2018, 26 de abril). Denuncia ambiental. "Mendoza es un pueblo sacrificable": el audio apocalíptico que se viralizó y causó pánico. Clarín. Recuperado de: https://www.clarin.com/sociedad/mendoza-pueblo-sacrificableaudio-apocaliptico-viralizo-causo-panico_0_ry80P016z.html

»Calalesina, A. (2021, 26 de junio). Fracking y sismos, un debate con datos. Más energía. Recuperado de: https://mase.Imneuquen.com/sismos/fracking-y-sismosun-debate-datos-n817804

»Callon, M., Lascoumes. P. y Barthe, Y. (2001). Agir dans un monde incertain. Essai sur la démocratie technique. París, Seuil. 
» Capogrossi, M.L. (2013). El prisma petrolero. Prácticas, memorias y discursos de los trabajadores de YPF en el Yacimiento Norte, Salta, Argentina. Taller, 2(2), 104-114.

" Chiappe, L. (2005). La Patagonia de pie. Ecología vs. Negociados. Chubut: Proyecto Lemu-Grupo de Amigos del Libro.

》Delgado, E. (2018). Fracking Vaca Muerta: Socioeconomic Implications of Shale Gas Extraction in Northern Patagonia, Argentina. Journal of Latin American Geography, 17(3), 102-131. DOI: https://doi.org/10.1353/lag.2018.0043

» Ferrante, S.B. y Giuliani, A. (2014). Hidrocarburos no convencionales en Vaca Muerta (Neuquén). ¿Recursos estratégicos para el autoabastecimiento energético en la Argentina del siglo XXI? Estado y Políticas Públicas, 3, 33-61.

» Freier, A. y Schaj, G. (2016). La fractura hidráulica en Argentina: Los cambios en el concepto de territorialidad y la emergencia de nuevos regímenes de soberanía. Enfoques, 14(25), 59-81.

》Funtowicz, S.O. y Ravetz, J.R. (1993). Science for the post-normal age. Futures, 25(7), 739-755.

» Gutiérrez Ríos, F., Wagner, L. y Giraud, M. (2021). Fracking en Argentina: consenso fósil y resistencias silenciadas. En O. Santos, A., L. Sánchez Vásquez y H. Escalante Moreno (Eds.), Minería y Extractivismos. Diálogos entre la academia y el activismo. Granada: Editorial de la Universidad de Granada. En prensa.

》 Gutierrez, R.A. e Isuani, F.J. (2014). La emergencia del ambientalismo estatal y social en Argentina. Revista de Administração Pública, 48(2), 295-322. DOI: https:// doi.org/10.1590/0034-76121700

» Hadad, M.G., Palmisano, T. y Wahren, J. (2020). Socio-territorial Disputes and Violence on Fracking Land in Vaca Muerta, Argentina. Latin American Perspectives, 48(1), 63-83. DOI: https://doi.org/10.1177/0094582X20975009

» Hess, J., Mannin, D.T., Iverson, T. y Cutler, H. (2019). Uncertainty, learning, and local opposition to hydraulic fracturing. Resource and Energy Economics, 55, 102-123. DOI: https://doi.org/10.1016/j.reseneeco.2018.11.001

» Instituto Geográfico Nacional (IGN), Ministerio de Defensa, Presidencia de la Nación (2020). Capas SIG. IGN. Recuperado de: https://www.ign.gob.ar/ NuestrasActividades/InformacionGeoespacial/CapasSIG

" Lis, A. y Stasik, A.K. (2017). Hybrid forums, knowledge deficits and the multiple uncertainties of resource extraction: Negotiating the local governance of shale gas in Poland. Energy Research \& Social Science, 28, 29-36. DOI: https://doi. org/10.1016/j.erss.2017.04.003

" Martín, F., Rojas, F. y Saldi, L. (2010). Domar el agua para gobernar. Concepciones socio-políticas sobre la naturaleza y la sociedad en contextos de consolidación del Estado provincial mendocinohacia finales del siglo XIX y principios del XX. Anuario del Centro de Estudios Históricos "Prof. Carlos S. A. Segreti", 10(10), 159-186.

》 Mendoza Invest (2016). Hidrocarburos. Agencia de Promoción de Inversiones, Gobierno de Mendoza. Recuperado de: http://invest.mendoza.gov.ar/?page_ id $=2649$

» Merlinsky, G. (2013). Introducción. La cuestión ambiental en la agenda pública. En G. Merlinsky (Coord.), Cartografías del conflicto ambiental en Argentina (pp. 1960). Buenos Aires: CLACSO-CICCUS. 
LUCRECIA WAGNER

》 Ministerio de Economía (s/f). Vaca Muerta. Argentina: Ministerio de Economía, República Argentina. Recuperado de: https://www.argentina.gob.ar/economia/ energia/vaca-muerta

» Montaña, E. (2007). Identidad Regional y Construcción Del Territorio En Mendoza (Argentina): Memorias y Olvidos Estratégicos. Bulletin de l'Institut Français d'études Andines, 36(2), 277-297. DOI: https://doi.org/10.4000/bifea.3908

" Montaña, E. (2008). Las disputas territoriales de una sociedad hídrica. Conflictos en torno al agua en Mendoza, Argentina. Revista Iberoamericana de Economía Ecológica, 9, 1-17. Recuperado de: http://www.iai.int/admin/site/sites/default/ files/uploads/PDF-de-REVIBEC.pdf

» Muñiz Terra, L. (2007). La privatización de la identidad petrolera: De la ilusión al desarraigo. AIBR, 2(1), 91-114. Recuperado de: http://sedici.unlp.edu.ar/ handle/10915/90784

» Natenzon, C. (2015). Presentación. En C. Natenzon y D. Ríos (Eds.), Riesgos, catástrofes y vulnerabilidades. Aportes desde la geografía y otras ciencias sociales para casos argentinos (pp. IX-XXV). Buenos Aires: Imago Mundi.

»Nievas, J.C. (2019). 3.2. Acciones judiciales frente al fracking en la provincia de Mendoza. En Informe Ambiental Anual 2019 (pp. 213-225). Buenos Aires: Fundación Ambiente y Recursos Naturales (FARN).

" O'Riordan, T. (2015). Fracking, Sustainability, and Democracy. Environment: Science and Policy for Sustainable Development, 57(1), 2-3. DOI: http://dx.doi.or $\mathrm{g} / 10.1080 / 00139157.2015 .985171$

" OPSur, Observatorio Petrolero Sur (2020, 17 de octubre). Receta repetida: subsidio a la extracción de gas sin horizonte de transición. Fundación de Investigaciones Sociales y Políticas (FISYP). Recuperado de: https://fisyp.org.ar/2020/10/17/ receta-repetida-subsidio-a-la-extraccion-de-gas-sin-horizonte-de-transicion-poropsur/

》Página12 (2016, 09 de noviembre). Denuncia de persecución. Página12. Recuperado de: https://www.pagina12.com.ar/2043-denuncia-de-persecucion

»Pellegrini, L. Arsel, M., Orta-Martínez, M. y Mena, C. F. (2020). International Investment Agreements, Human Rights, and Environmental Justice: The Texaco/ Chevron Case from the Ecuadorian Amazon. Journal of International Economic Law, 23(2), 455-468. DOI: https://doi.org/10.1093/jiel/jgaa016

"Perez, A. (2018, 24 de mayo). Ataque a la libertad de expresión. Mendoza: diputados oficialistas denuncian penalmente a docente por enviar audio contra el fracking. La Izquierda Diario. Recuperado de: https://www.laizquierdadiario.com/ Mendoza-diputados-oficialistas-denuncian-penalmente-a-docente-por-enviaraudio-contra-el-fracking

》Prieto, M. del R., Rojas, F., Martín, F., Araneo, D., Villalba, R., Rivera, J.A. y Gil Guirado, S. (2020). Sequías extremas en Mendoza durante el siglo XX y principios del XXI. Administración de la carencia y conflictos socio-políticos. En A. Zarrilli y M. Ruffini (Comps.), Medio Ambiente y transformación rural en la Argentina contemporánea. Bernal: Universidad Nacional de Quilmes. En prensa.

» Radovich, J.C. (2017). Fractura hidráulica y conflicto territorial en la comunidad mapuche Campo Maripe, Neuquén, Argentina. Revista GeoPantanal, 12(22), 89-104. Recuperado de: https://periodicos.ufms.br/index.php/revgeo/article/ view/3806 
» Riffo, L. (2017). Fracking and Resistance in the Land of Fire. NACLA Report on the Americas, 49(4), 470-475. DOI: https://doi.org/10.1080/10714839.2017.1409376

» Río Negro (2013, 28 de noviembre). Allen había prohibido el fracking, el STJ dijo no. Río Negro. Recuperado de: https://www.rionegro.com.ar/allen-habia-prohibidoel-fracking-el-stj-dijo-no-KPRN_1388628/

»Rosa, L. y D'Odorico, P. (2019). The water-energy-food nexus of unconventional oil and gas extraction in the Vaca Muerta Play, Argentina. Journal of Cleaner Production, 207, 743-750. DOI: https://doi.org/10.1016/j.jclepro.2018.10.039

»Sales, T., Giusiano, A., Gutiérrez Schmidt, N., Alonso, J. C. y Muñoz, O. (2014). Controles estratigráficos sobre propiedades de la roca en los shales de la formación Vaca Muerta, cuenca neuquina, provincia de Neuquén. IX Congress on Exploration and Development of Hydrocarbons. Mendoza, Argentina.

»Salomone, M. (2021). El fracking en Mendoza, una iniciativa al extremo de lo posible. Perfiles Latinoamericanos, 29(57). DOI: https://doi.org/10.18504/pl2957004-2021

»Salomone, M. (2019, 20 de mayo). Vicisitudes de un audio viral en el debate público sobre el fracking. Unidiversidad. Recuperado de: https://www. unidiversidad.com.ar/vicisitudes-de-un-audio-viral-en-el-debate-publico-sobre-elfracking

》Saulino, F. (2018). Argentina: Energy Extraction and Communities: Can Shale Development Proceed without Causing Pollution and Conflicts? En S. GamperRabindran (Ed.), The Shale Dilemma. A Global Perspective on Fracking and Shale Development (305-341). Pittsburgh: Pittsburgh University Press.

» Serrano Narváez, H. (2013). Caso Chevron-Texaco: cuando los pueblos toman la palabra. Quito: Universidad Andina Simón Bolívar, Sede Ecuador-Corporación Editora Nacional.

"Svampa, M. y Antonelli, M. (2009). Minería transnacional, narrativas del desarrollo y resistencias sociales. Buenos Aires: Biblos.

"Svampa, M. y Viale, E. (2014). Maldesarrollo. La Argentina del extractivismo y el despojo. Buenos Aires: Katz Editores.

"Trifogli, V. (2020, 09 de diciembre). Sismos en Añelo, una analogía con el nuevo coronavirus. Río Negro. Recuperado de: https://www.rionegro.com.ar/sismos-enanelo-una-analogia-con-el-nuevo-coronavirus-1394721/

» U.S. Geological Survey (2003). SRTM-3 DEM NASA/NIMA 2003, USGS. Recuperado de: http://earthexplorer.usgs.gov

》 Unidiversidad (2019, 19 de diciembre). Duro comunicado del lanigla-Conicet contra la reforma de la 7722. Unidiversidad. Recuperado de: https://www. unidiversidad.com.ar/duro-comunicado-del-ianigla-conicet-contra-la-reforma-dela-7722

»Wagner, L. (2019). Agricultura, cultura del oasis y megaminería en Mendoza. Debates y disputas. Mundo Agrario, 20(43). DOI: https://doi. org/10.24215/15155994e106

»Wagner, L. y Giraud, M. (2011). El proyecto minero Potasio Río Colorado: Conflicto socioambiental, impactos regionales y falta de integralidad en la evaluación ambiental. En H. Alimonda (Coord.), La Naturaleza colonizada: Ecología política y minería en América Latina (pp. 261-288). Buenos Aires: CLACSO. 
Fracking en el sur de Mendoza: riesgos...

»Wagner, L. y Giraud, M. (2013). "Sin licencia social no hay minería": Incertidumbre científica, resistencia social y debate político generados por la megaminería en Mendoza". Entramados y Perspectivas, 3(3), 91-120.

》Wagner, L. y Walter, M. (2020). Cartografía de la conflictividad minera en argentina (2003-2018). Un análisis desde el Atlas de Justicia Ambiental. En G. Merlinsky (Comp.), Cartografías del conflicto ambiental en Argentina 3 (pp. 247-278). Buenos Aires: CICCUS-CLACSO.

》Wagner, L., Maurtua Konstantinidis, E. y Blanco, G. (2018). Aspectos socioambientales de la transición energética en la Argentina. Policy Paper. Buenos Aires: FARN

»Williams, L. y Sovacool, B. (2019). The discursive politics of 'fracking': Frames, storylines, and the anticipatory contestation of shale gas development in the United Kingdom. Global Environmental Change, 58. DOI: https://doi.org/10.1016/j. gloenvcha.2019.101935

»Wyczykier, G. y Acacio, J. A. (2021). Sobre promesas, esperanzas públicas y resistencias sociales en las tierras del fracking: Vaca Muerta en cuestión. e-l@ tina, 19(74), 62-87. Recuperado de: https://publicaciones.sociales.uba.ar/index. php/elatina/article/view/6270

\section{Lucrecia Wagner / lucrewagner@gmail.com}

Licenciada en Diagnóstico y Gestión Ambiental (Universidad Nacional del Centro de la Provincia de Buenos Aires) y Doctora en Ciencias Sociales y Humanas (Universidad Nacional de Quilmes). Investigadora Adjunta del CONICET, Argentina, y docente del Doctorado en Ciencias Sociales de la Universidad Nacional de Cuyo. Publicó artículos en revistas de la especialidad sobre conflictos ambientales y procedimientos de evaluación de impacto ambiental de proyectos extractivos, con especial énfasis en la legislación ambiental y los mecanismos de participación social. 